Title page

\title{
Dental attendance and behavioural pathways to adult oral health inequalities
}

\section{Authors:}

Carol C Guarnizo-Herreño ${ }^{a}$, Shaun Scholes ${ }^{b}$, Anja Heilmann ${ }^{b}$, Rhiannon C O'Connor ${ }^{c}$, Elizabeth Fuller $^{d}$, Jing Shen ${ }^{\mathrm{e}}$, Richard G Watt ${ }^{\mathrm{b}}$, Stephen Morris ${ }^{\mathrm{f}}$, John Wildman ${ }^{\mathrm{g}}$, Georgios Tsakos ${ }^{\mathrm{b}}$

a Departamento de Salud Colectiva, Facultad de Odontología Universidad Nacional de Colombia, Bogotá, Colombia

${ }^{\mathrm{b}}$ Department of Epidemiology and Public Health University College London, London, United Kingdom

'School of Dental Sciences, Newcastle University, Newcastle Upon Tyne, United Kingdom

${ }^{\mathrm{d}}$ National Centre for Social Research NatCen, London, United Kingdom

e GlaxoSmithKline, Belgium and Population Health Sciences Institute Newcastle University, Newcastle Upon Tyne, United Kingdom

(Although Jing Shen works now for GSK, the work associated with the paper was conducted during her time at Newcastle University)

${ }^{f}$ Cambridge Centre for Health Services Research

University of Cambridge, Cambridge, United Kingdom

${ }^{\mathrm{g}}$ Population Health Sciences Institute

Newcastle University, Newcastle Upon Tyne, United Kingdom

Corresponding author:

Carol C. Guarnizo-Herreño

Carrera 30 No. 45-03, Facultad de Odontología | Bogotá, Colombia

Tel/Fax: +57 (1) 3165000 Ext. 16019 | Email: ccguarnizoh@unal.edu.co

Total Word Count: 2,997 
50

51

\section{Abstract}

Background: While inequalities in oral health are documented, little is known about the extent to which they are attributable to potentially modifiable factors. We examined the role of behavioural and dental attendance pathways in explaining oral health inequalities among adults in England, Wales and Northern Ireland.

Methods: Using nationally representative data, we analysed inequalities in self-rated oral health and number of natural teeth. Highest educational attainment, equivalised household income and occupational social class were used to derive a latent socioeconomic position (SEP) variable. Pathways were dental attendance and behaviours (smoking and oral hygiene). We used structural equation modelling to test the hypothesis that SEP influences oral health directly and also indirectly via dental attendance and behavioural pathways.

Results: Lower SEP was directly associated with fewer natural teeth and worse self-rated oral health (standardized path coefficients, -0.21 ( $\mathrm{SE}=0.01$ ) and $-0.10(\mathrm{SE}=0.01$ ) respectively). We also found significant indirect effects via behavioural factors for both outcomes and via dental attendance primarily for self-rated oral health. While the standardized parameters of total effects were similar between the two outcomes, for number of teeth the estimated effect of SEP was mostly direct while for self-rated oral health it was almost equally split between direct and indirect effects.

Conclusion: Reducing inequalities in dental attendance and health behaviours is necessary but not sufficient to tackle socioeconomic inequalities in oral health. 


\section{Introduction}

Inequalities in oral health exist in different countries with worse oral health for those in lower socioeconomic position (SEP). 1,2 These unfair and potentially avoidable differences are linked to systematic social disadvantage and poorer access to resources. 3 Socioeconomic inequalities in both general and oral health have persisted during the last decades and even increased for certain outcomes. ${ }^{4,5}$ While there is ample evidence documenting oral health inequalities, less is known about the pathways explaining them, and this is essential to inform policy.

Different interlinked pathways have been suggested to explain the socially patterned distribution of oral health. ${ }^{6}$ Behaviours can potentially explain oral health inequalities as they differ according to SEP 7,8 and could be influenced by stressful living or working conditions and differential access to material resources such as dental services. ${ }^{6}$ Studies show that dental attendance or oral health behaviours do not fully explain inequalities and their role might change according to the context and age. ${ }^{9-11}$ Looking at general health, the role of behaviours in explaining the socioeconomic gradient in cardiometabolic disorders and mortality might vary according to population and setting. ${ }^{12}$ Furthermore, a recent systematic review showed that material, psychosocial and behavioural factors contribute to explaining socioeconomic inequalities in self-rated health, with material factors (e.g., crowding, poor housing conditions or financial difficulties) being more important given their larger independent effects and their effects through psychosocial and behavioural factors. ${ }^{13}$

In the UK, a study using Adult Dental Health Survey (ADHS) 1998 data showed a significant role for dental attendance patterns and barriers alone in explaining inequalities in the number of sound teeth. ${ }^{14}$ However, no studies have used the most recent nationally representative data (ADHS 2009) and simultaneously analyse the extent to which oral health inequalities may be attributable to potentially modifiable factors such as behaviours and dental care, without viewing them in isolation, by employing a structural equation modelling (SEM) framework that allows comparisons between pathways by quantifying direct and indirect effects. ${ }^{15}$ Given the persistent oral health inequalities 16,17 in this population, such knowledge has clear implications for the focus and balance of public health policies to address these inequalities. 
115 Therefore, we examined the role of behavioural and dental attendance pathways in 116 explaining socioeconomic inequalities in self-rated oral health and clinically assessed number 117 of teeth, using nationally representative data and a SEM analytical approach.

118

119

120

121

122

\section{Methods}

Figure 1 presents the conceptual model that informed our analyses. We hypothesized that SEP would influence oral health directly and also indirectly via dental attendance and behaviours.

\section{Data Source and Study Sample}

We analysed ADHS 2009 data, which employed a two-stage cluster and probabilistic sample design to provide representative samples of individuals aged 16 years and over in England, Wales and Northern Ireland (Scotland did not participate). All adults within selected households were approached for an interview and those with at least one natural tooth were also invited to a clinical dental examination. Interview data were obtained from a sample of 11,380 individuals, of which 6,469 completed the clinical examination. The household response rate was $60 \%$, and the individual response rate within households was $84 \%$. Ethical approval for the ADHS 2009 was granted by the Oxford B Research Ethics Committee. ${ }^{18}$

We included in the analyses participants aged 21 years and older to ensure accurate data on highest educational attainment. The sample was also limited to participants with complete data on the employed variables ( $85 \%$ of the initial sample). Since information on number of teeth was obtained from the clinical examination, only dentate adults who completed the examination were included in that analysis. This led to analytical samples of 8,030 for selfrated oral health and 5,193 for number of teeth.

\section{Variables}

\section{Outcomes}

We used one subjective and one clinical oral health outcome. Self-rated oral health was assessed via the question 'would you say your dental health (mouth, teeth and/or dentures) 
is...' with response categories: very good, good, fair, bad, and very bad. This widely used subjective outcome reflects people's current perception of their oral health and is associated with clinical measures and unmet treatment needs. ${ }^{19-21}$ The variable was coded so that higher scores indicate better oral health perception. On the other hand, clinically assessed number of natural teeth is a measure of life-time oral health that captures the cumulative effect of oral diseases and experience of dental treatment. ${ }^{1,22,23}$ It has been linked to important functions such as eating and socializing. ${ }^{24}$

Socioeconomic position (SEP)

We employed three indicators to derive the latent SEP: highest educational attainment, equivalised household income, and occupational social class. Educational attainment was categorised into no qualifications, qualifications below degree level, and degree level. Household income was adjusted for household size and composition ${ }^{25}$ and recoded to quintiles. For occupational social class, we used the UK three-category National Statistics Socio-Economic Classification scheme (NS-SEC) which allocates people to managerial and professional, intermediate, and routine-manual occupations. ${ }^{26}$ We included an additional category of those who never worked or were long-term unemployed. All indicators were recoded such that higher values on the latent variable represent lower SEP.

\section{Mediators - explanatory pathways}

Dental attendance

We used the dental attendance pattern (regular check-ups vs occasionally or only when having trouble), frequency of dental visits (longer than every 2 years, every 2 years, every year, or every 6 months), and time since last dental visit (over 12 months, within 7-12 months, or within 6 months) to define a latent dental attendance variable that captures access and use of oral health services. Higher scores on the dental attendance latent variable represent participants who visit the dentist more often and for regular check-ups. For the outcome of number of teeth, our conceptual model was not identified and therefore, based on the model's empirical testing, we used the observable characteristic for the dental attendance pattern instead of the latent variable. 
Oral health behaviours

180 Two behavioural variables were considered: smoking (current smoker, past smoker, or never smoked) and oral hygiene (tooth brushing frequency: more than twice daily, twice daily, once daily, or less often). They were coded with lower values indicating healthier behaviours and were entered independently in analyses because their internal consistency when trying to create a latent variable was very low (Cronbach's alpha=0.103). Sugar consumption was not included in analyses since diet related questions were not comprehensive to provide an accurate sugar consumption measure.

\section{Statistical analysis}

To test our model, we used structural equation modelling (SEM) with the asymptotic distribution free method, as our models included categorical variables. ${ }^{27}$ In the first stage, the two latent variables (SEP and dental attendance) were specified separately using Confirmatory Factor Analytic models, where all standardized factor loadings were above the benchmark of 0.4 (from 0.60 to 0.95 ). The second stage involved fitting the path analytic models, which included the latent constructs, to jointly estimate the direct and indirect associations of SEP with the outcomes. We derived standardized model parameters so that their relative sizes can be compared. Age group (21-34, 35-49, 50-64, 65+ years) was also accounted for given its association with oral health and SEP.

The Comparative Fit Index (CFI), Root Mean Square Error of Approximation (RMSEA) and Standardized Root Mean Square Residual (SRMR) were used to assess goodness of fit, with CFI $>0.95$ and RMSEA and SRMR $<0.05$ indicating good model fit. ${ }^{28,29}$ Dental attendance and behavioural variables error terms were allowed to correlate as that improved the model fit, possibly reflecting the presence of unobserved factors associated with both pathways. All analyses were conducted in Stata 15 . We estimated un-weighted models since the asymptotic distribution free method with the sem function in Stata does not allow for use of weights. In sensitivity analysis, we specified sem with a maximum likelihood method which allowed considering the appropriate survey weights, and estimates were almost identical with the main findings (Appendix Figure 1) indicating that our results were robust to complex sampling design and survey weights. In other sensitivity analysis, models were run separately by gender 
and two age groups (21-49 and 50+ years). As most excluded participants from our completecase analyses had missing data on income, we also estimated the models with missing income data imputed using two approaches: Bayesian multiple imputation and simple regression techniques.

Results

217 Descriptive statistics are presented in Appendix Table 1. Over two thirds of adults rated their oral health as good or very good, while dentate participants had on average $25.6(S D=5.7)$ natural teeth.

The path analytic models (Figures 2 and 3 for self-rated oral health and number of teeth respectively) had satisfactory goodness of fit; the SRMR was 0.022 for the self-rated oral health model and 0.027 for the number of teeth model. The respective CFIs were 0.972 and 0.961 and the RMSEAs were 0.040 and 0.049 .

In both models, lower SEP was associated with higher levels of unhealthy behaviours (smoking, oral hygiene) and less favourable dental attendance pattern. The mediating variables were also associated with the outcomes in the expected direction.

SEP was associated with both outcomes (Table 1). The direct associations of SEP on both outcomes were significant, with standardized path coefficients of -0.10 (SE=0.01) for selfrated oral health and $-0.21(\mathrm{SE}=0.01)$ for number of teeth. Since these are standardized regression coefficients, they can be compared, showing that the direct influence of SEP was twice as large on number of teeth than on self-rated oral health. In practical terms, the -0.21 coefficient for number of teeth means that for one SD lower SEP according to the latent variable, there would be around 1.15 fewer teeth on average, i.e. a decrease in the mean number of teeth from 25.6 to 24.45. Putting this in context, and considering that 32 teeth constitutes the full natural dentition, on average, adults had lost 6.4 teeth, and therefore an additional loss of 1.15 teeth reflects approximately a further $18 \%$ tooth loss (in relative terms) that would be due to SEP alone (as these estimates refer to direct effects only). 
We also found significant indirect associations between SEP and the outcomes via behavioural factors and dental attendance. The indirect association was more modest for number of teeth where $84 \%$ of the estimated total effect of SEP was direct and $16 \%$ indirect. For self-rated oral health, $52 \%$ of the estimated total effect of SEP was direct and $48 \%$ indirect. The main difference between the two outcomes was in the dental attendance pathway which played a stronger role in explaining inequalities in self-rated oral health (indirect effect: $-0.054 ; 95 \%$ $\mathrm{Cl}:-0.062,-0.045)$ than in number of teeth (indirect effect: $-0.013 ; 95 \% \mathrm{Cl}:-0.019,-0.007$ ) (Table 1).

Estimating the models after imputing the missing income data led to almost identical results as for complete case analysis (Appendix Figure 2). We also ran the model for self-rated oral health only among dentate participants who completed the clinical examination and found almost identical estimates with the aforementioned results (Appendix Figure 3). When models were run separately for 21-49-year-olds and 50+ year-olds, the indirect association between SEP and number of teeth via smoking was larger among younger compared to older adults (Appendix Figure 4). The only model showing a predominant indirect effect of SEP on oral health was for self-rated oral health among older adults ( $57 \%$ indirect effect) and this was primarily via dental attendance (Appendix Figure 4). Models stratified by gender showed similar estimates for men and women (Appendix Figure 5). We also included gender in the main models and results were virtually identical to those reported above.

\section{Discussion}

This analysis showed that SEP had a predominant direct effect on oral health, with lower SEP associated with worse self-rated oral health and fewer natural teeth. The overall explanation of inequalities was comparable between the two outcomes (standardized parameters for total effects of SEP on outcomes: -0.19 and -0.25$)$; however, SEP affected the number of teeth mostly directly (i.e. not operating through health behaviours or dental attendance), while for self-rated oral health it was almost equally split between direct and indirect effects. The behavioural pathways (smoking and oral hygiene) had an overall modest role in explaining socioeconomic inequalities in both outcomes, although these indirect associations were 
273 relatively larger for self-rated oral health. The dental attendance pathway also played a

274 minimal role for number of teeth, but a much stronger role for inequalities in self-rated oral

275 health. Overall, the indirect effects of SEP through behaviours and dental attendance were stronger for self-rated oral health, a subjective measure of current oral health.

277

278 In this analysis, the direct path from SEP to dental attendance indicates that adults in lower SEP visit the dentist less often and are less likely to go for regular check-ups, despite having poorer self-rated oral health. Potential reasons for this pattern include financial barriers, geographical barriers, dental anxiety and perceptions and beliefs about oral health and dental care. ${ }^{30,31}$ Understanding and addressing these issues could shed more light on the complex pathways leading to inequalities in perceived oral health.

Our results corroborate those from Australia ${ }^{32}$ in showing a limited role for dental attendance in explaining inequalities in number of teeth. This implies that other mechanisms may play a greater role for these inequalities, even under different dental care systems. This, however, is also influenced by the context, as considerable proportions of socioeconomic inequality in number of teeth were attributable to dental attendance in certain European countries, but not others. ${ }^{9}$ Considering the UK context and current analyses, dental attendance seems to have a generally modest influence on socioeconomic inequalities in number of teeth among dentate adults.

The overall larger direct effects of SEP on number of teeth, compared to self-rated oral health, could be partly due to the nature of exposure and outcomes employed in this study. The SEP indicators were either acquired early in life (highest educational attainment) or a result of accumulating years of affluence (income and occupational class), therefore better suited to a cumulative measure of disease and treatment over the life course (number of teeth) than on a current oral health rating. Moreover, the predominantly direct effects indicate the potential importance of the unequal distribution of wealth and broader social structures for addressing inequalities.

303 Our findings also agree with evidence showing that behaviours alone have an overall limited 304 role in explaining inequalities in oral health, ${ }^{11,33}$ and general health. ${ }^{13,34,35}$ This highlights the 
need for direct action on more upstream structural determinants of health. Among the examined behaviours, smoking showed relatively larger effects, in line with its role in partially explaining inequalities in other non-communicable diseases, therefore being a potentially relevant intervention point. ${ }^{36,37}$ Health promotion strategies addressing the underlying social and commercial determinants of smoking could make a considerable contribution to both population health and reducing inequalities.

We could not explore how the pathways to oral health inequalities operate across the life course as we analysed cross-sectional data. However, age stratified models suggest that behaviours, particularly smoking, could have a larger role earlier in adulthood, while dental attendance seems to be more relevant among older adults. The variation in these associations by different age groups suggests that pathways to oral health inequalities may be dynamic across adulthood or vary across generations.

This study has limitations related to variable selection and data availability. First, the potentialy important psychosocial pathway could not be assessed due to lack of relevant information. Second, limited information on material factors and other relevant behaviours such as sugar consumption did not allow us to assess their role in explaining oral health inequalities. Third, the incomplete household income data led to some higher income households being placed in lower income brackets. This means that our results provide, if anything, an underestimation of the actual associations. Fourth, behaviours and dental attendance data were self-reported which could introduce response bias, particularly social desirability bias. This could underestimate the role of these factors in explaining oral health inequalities. Finally, the use of cross-sectional data makes it impossible to establish a temporal sequence, an important issue when analysing potential mediators, or to rule out a potential cohort effect when comparing age groups. This issue might be particularly relevant when analysing number of teeth, a cumulative measure of oral health. Nevertheless, oral health behaviours and dental attendance patterns seem relatively stable over time, ${ }^{38}$ somewhat partly mitigating the impact of this limitation on the study findings.

Thinking about the strengths, we used nationally representative data and a SEM analytical approach to test a theoretical model of pathways to socioeconomic inequalities in oral health 
of adults in England, Wales and Northern Ireland. The SEM method allowed quantification of the direct and indirect effects of SEP on oral health and comparisons between different mediating pathways. To our knowledge, this is one of very few studies that quantified such effects using data from a national survey and employing two oral health outcomes and different but related SEP indicators.

In conclusion, this study showed that the pathways to oral health inequalities are complex. Socioeconomic position had mostly a direct effect on the oral health of adults in England, Wales and Northern Ireland, with the indirect effects varying according to the outcome analysed. Behavioural and dental attendance factors accounted for about half of the inequalities in self-rated oral health, whereas a stronger direct effect of SEP was observed on number of teeth. Policy makers should bear in mind that, improving access and use of dental care services and promoting healthy behaviours (e.g. smoking cessation) among those in lower socioeconomic groups may reduce oral health inequalities to some extent, but those interventions alone will not successfully eliminate inequalities. While behavioural interventions are still relevant, mostly in terms of inequalities in perceived oral health, their potential impact -if applied in isolation- on inequalities in clinical oral health is much less profound. This shifts the public health emphasis towards the structural determinants of health and points to policies that address the unequal distribution of wealth and privilege as being necessary to improve the unequal distribution of oral health across socioeconomic groups. A public health approach that looks at inequalities impact assessment in all policies may be a good step forward. 
- While there is substantial evidence documenting socioeconomic inequalities in oral health,

371 less is known about the pathways explaining these inequalities.

372 - Understanding the mechanisms behind socioeconomic inequalities in oral health is essential

373 to inform policy development.

374 - However, no study has simultaneously assessed the role of dental attendance and

375 behavioural mechanisms by employing a structural equation modelling framework in a nationally representative sample.

\section{What this study adds?}

- Lower socioeconomic position (SEP) was associated with fewer natural teeth through a predominantly direct effect, while dental attendance and behaviours accounted for a very small part of this association.

- Lower SEP was also associated with worse self-rated oral health, with almost equal direct and indirect (through behaviours and dental attendance) effects.

- The pathways to oral health inequalities are complex and vary across different outcomes. Improving access to and use of dental care services and promoting healthy behaviours among those in lower socioeconomic groups may reduce oral health inequalities to some extent, primarily in relation to self-rated oral health though not equally so for number of teeth. However, those interventions alone will not successfully eliminate inequalities.

Acknowledgments: We want to acknowledge the considerable contribution of Professor Jimmy Steele in the original planning of this work. The drafting of the paper was finalized after his death and therefore he bears no responsibility for the final version.

Authors' contribution: All authors meet the ICMJE authorship criteria. CGH contributed to conception, design, analysis and interpretation, drafted and critically revised the manuscript; SS contributed to analysis and interpretation, and critically revised the manuscript; $A H, R O^{\prime} C$, $E F, J S, R G W, S M$ critically revised the manuscript; JW contributed to data acquisition, analysis and interpretation, and critically revised the manuscript; GT contributed to conception, 
400

401

402

403

404

405

406

407

408

409

410

411

412

413

414

415

416

417

418

419

420

421

422

423

424

425

426

427

428

429

430

431

432

433

434

435

436

437

438

439

440

design, analysis and interpretation, and critically revised the manuscript. All authors approved the final manuscript.

Funding: This work was supported by the UK Economic and Social Research Council [Grant Number ES/K004689/1] as part of the Secondary Data Analysis Initiative.

Competing interests: None declared

Patient consent: Not required for this study. Ethical approval for the ADHS 2009 was granted by the Oxford B Research Ethics Committee.

\section{References}

1. Celeste RK, Nadanovsky P, Ponce de Leon A, Fritzell J. The individual and contextual pathways between oral health and income inequality in Brazilian adolescents and adults. Social Science and Medicine. 2009;69(10):1468-1475.

2. Schwendicke F, Dorfer CE, Schlattmann P, Page LF, Thomson WM, Paris S. Socioeconomic inequality and caries: a systematic review and meta-analysis. Journal of Dental Research. 2015;94(1):10-18.

3. Marmot M, Friel S, Bell R, Houweling TAJ, Taylor S, Commission on Social Determinants of Health. Closing the gap in a generation: health equity through action on the social determinants of health. Lancet. 2008;372(9650):1661-1669.

4. Bleich SN, Jarlenski MP, Bell CN, LaVeist TA. Health inequalities: trends, progress, and policy. Annu Rev Public Health. 2012;33:7-40.

5. Do LG, Spencer AJ, Slade GD, Ha DH, Roberts-Thomson KF, Liu P. Trend of incomerelated inequality of child oral health in Australia. J Dent Res. 2010;89(9):959-964.

6. Bartley M. Health inequality: an introduction to theories, concepts, and methods. Cambridge: Polity Press; 2004.

7. Singh A, Rouxel P, Watt RG, Tsakos G. Social inequalities in clustering of oral health related behaviors in a national sample of British adults. Prev Med. 2013;57(2):102-106.

8. Kino S, Bernabe E, Sabbah W. Socioeconomic inequality in clusters of health-related behaviours in Europe: latent class analysis of a cross-sectional European survey. BMC Public Health. 2017;17(1):497.

9. Shen J, Listl S. Investigating social inequalities in older adults' dentition and the role of dental service use in 14 European countries. Eur J Health Econ. 2018;19(1):45-57.

10. Guarnizo-Herreno CC, Watt RG, Garzon-Orjuela N, Tsakos G. Explaining oral health inequalities in European welfare state regimes: The role of health behaviours. Community Dent Oral Epidemiol. 2019;47(1):40-48.

11. Sabbah W, Tsakos G, Sheiham A, Watt RG. The role of health-related behaviors in the socioeconomic disparities in oral health. Social Science and Medicine. 2009;68(2):298303. 
12. Petrovic $D$, de Mestral $C$, Bochud $M$, et al. The contribution of health behaviors to socioeconomic inequalities in health: A systematic review. Prev Med. 2018;113:15-31.

13. Moor I, Spallek J, Richter M. Explaining socioeconomic inequalities in self-rated health: a systematic review of the relative contribution of material, psychosocial and behavioural factors. J Epidemiol Community Health. 2017;71(6):565-575.

14. Donaldson AN, Everitt B, Newton T, Steele J, Sherriff M, Bower E. The effects of social class and dental attendance on oral health. Journal of Dental Research. 2008;87(1):6064.

15. Ploubidis GB, Destavola BL, Grundy E. Health differentials in the older population of England: an empirical comparison of the materialist, lifestyle and psychosocial hypotheses. BMC Public Health. 2011;11:390.

16. Steele J, Shen J, Tsakos G, et al. The Interplay between socioeconomic inequalities and clinical oral health. J Dent Res. 2015;94(1):19-26.

17. Guarnizo-Herreno CC, Watt RG, Fuller E, et al. Socioeconomic position and subjective oral health: findings for the adult population in England, Wales and Northern Ireland. BMC Public Health. 2014;14:827.

18. O'Sullivan I, Lader D, Beavan-Seymour C, Chenery V, Fuller E, Sadler K. Foundation Report: Adult Dental Health Survey 2009 (Technical information). NHS Information Centre. 2011.

19. Locker D, Mscn EW, Jokovic A. What do older adults' global self-ratings of oral health measure? J Public Health Dent. 2005;65(3):146-152.

20. Pattussi MP, Peres KG, Boing AF, Peres MA, da Costa JSD. Self-rated oral health and associated factors in Brazilian elders. Community Dentistry and Oral Epidemiology. 2010;38(4):348-359.

21. Benyamini $Y$, Leventhal $H$, Leventhal EAEA. Self-rated oral health as an independent predictor of self-rated general health, self-esteem and life satisfaction. Social Science and Medicine. 2004;59(5):1109-1116.

22. Aida J, Kondo K, Kondo N, Watt RG, Sheiham A, Tsakos G. Income inequality, social capital and self-rated health and dental status in older Japanese. Social Science and Medicine. 2011;73(10):1561-1568.

23. Bernabe E, Marcenes W. Income inequality and tooth loss in the United States. Journal of Dental Research. 2011;90(6):724-729.

24. Sheiham A, Steele JG, Marcenes W, Finch S, Walls AW. The impact of oral health on stated ability to eat certain foods; findings from the National Diet and Nutrition Survey of Older People in Great Britain. Gerodontology. 1999;16(1):11-20.

25. McClements LD. Equivalence scales for children. J Public Econ. 1977;8(2):191-210.

26. Chandola T. Social class differences in mortality using the new UK National Statistics Socio-Economic Classification. Social Science and Medicine. 2000;50(5):641-649.

27. Browne MW. Asymptotically distribution-free methods for the analysis of covariance structures. Br J Math Stat Psychol. 1984;37(1):62-83.

28. Hu Lt, Bentler PM. Cutoff criteria for fit indexes in covariance structure analysis: Conventional criteria versus new alternatives. Structural Equation Modeling: $A$ Multidisciplinary Journal. 1999;6(1):1-55.

29. Hooper D, Coughlan J, Mullen MR. Structural equation modelling: Guidelines for determining model fit. Electronic journal of business research methods. 2008;6(1):5360 . 
30. Hakeberg $\mathrm{M}$, Wide Boman U. Dental care attendance and refrainment from dental care among adults. Acta Odontol Scand. 2017;75(5):366-371.

31. Listl S, Moeller J, Manski R. A multi-country comparison of reasons for dental nonattendance. Eur J Oral Sci. 2014;122(1):62-69.

32. Sanders AE, Spencer AJ, Slade GD. Evaluating the role of dental behaviour in oral health inequalities. Community Dentistry and Oral Epidemiology. 2006;34(1):71-79.

33. Polk DE, Weyant RJ, Manz MC. Socioeconomic factors in adolescents' oral health: are they mediated by oral hygiene behaviors or preventive interventions? Community Dent Oral Epidemiol. 2010;38(1):1-9.

34. Hiyoshi A, Fukuda Y, Shipley MJ, Brunner EJ. Health inequalities in Japan: the role of material, psychosocial, social relational and behavioural factors. Soc Sci Med. 2014;104:201-209.

35. Giesinger I, Goldblatt P, Howden-Chapman P, Marmot M, Kuh D, Brunner E. Association of socioeconomic position with smoking and mortality: the contribution of early life circumstances in the 1946 birth cohort. Journal of Epidemiology and Community Health. 2014;68(3):275-279.

36. Floud S, Balkwill A, Moser $\mathrm{K}$, et al. The role of health-related behavioural factors in accounting for inequalities in coronary heart disease risk by education and area deprivation: prospective study of 1.2 million UK women. BMC Med. 2016;14(1):145.

37. Stringhini $S$, Zaninotto $P$, Kumari $M$, Kivimäki $M$, Lassale $C$, Batty GD. Socio-economic trajectories and cardiovascular disease mortality in older people: the English Longitudinal Study of Ageing. Int J Epidemiol. 2018;47(1):36-46.

38. Netuveli G, Sheiham A, Watt RG. Does the 'inverse screening law' apply to oral cancer screening and regular dental check-ups? J Med Screen. 2006;13(1):47-50.

39. Preacher KJ, Hayes AF. Asymptotic and resampling strategies for assessing and comparing indirect effects in multiple mediator models. Behav Res Methods. 2008;40(3):879-891. 
528 Table 1 - Standardized parameters with their 95\% confidence intervals for direct, indirect, and total effects 538 of SEP on oral health

\begin{tabular}{|c|c|c|c|c|}
\hline & \multicolumn{4}{|c|}{ Outcome } \\
\hline & \multicolumn{2}{|c|}{ Good self-rated oral health } & \multicolumn{2}{|l|}{ Number of teeth } \\
\hline & $\begin{array}{c}\text { Standardized parameter } \\
(95 \% \mathrm{Cl})\end{array}$ & $\%$ & $\begin{array}{c}\text { Standardized parameter } \\
\qquad(95 \% \mathrm{CI})\end{array}$ & $\%$ \\
\hline Direct & $-0.099(-0.123,-0.075)$ & $51.8 \%$ & $-0.209(-0.238,-0.181)$ & $83.8 \%$ \\
\hline Indirect via smoking & $-0.026(-0.032,-0.020)$ & & $-0.019(-0.024,-0.014)$ & \\
\hline Indirect via cleaning & $-0.013(-0.017,-0.009)$ & & $-0.009(-0.014,-0.004)$ & \\
\hline $\begin{array}{l}\text { Indirect via dental } \\
\text { attendance }^{\text {a }}\end{array}$ & $-0.054(-0.062,-0.045)$ & & $-0.013(-0.019,-0.007)$ & \\
\hline Overall indirect & & $48.2 \%$ & & $16.2 \%$ \\
\hline Total & $-0.191(-0.214,-0.168)$ & & $-0.250(-0.279,-0.221)$ & \\
\hline
\end{tabular}

531 Note: negative coefficients indicate that lower SEP is associated with poorer self-rated oral health. Confidence 532 intervals were obtained using bootstrapping procedure via 1000 iterations. ${ }^{39}$

533 a Pattern of dental attendance for the outcome of number of teeth

534

535 


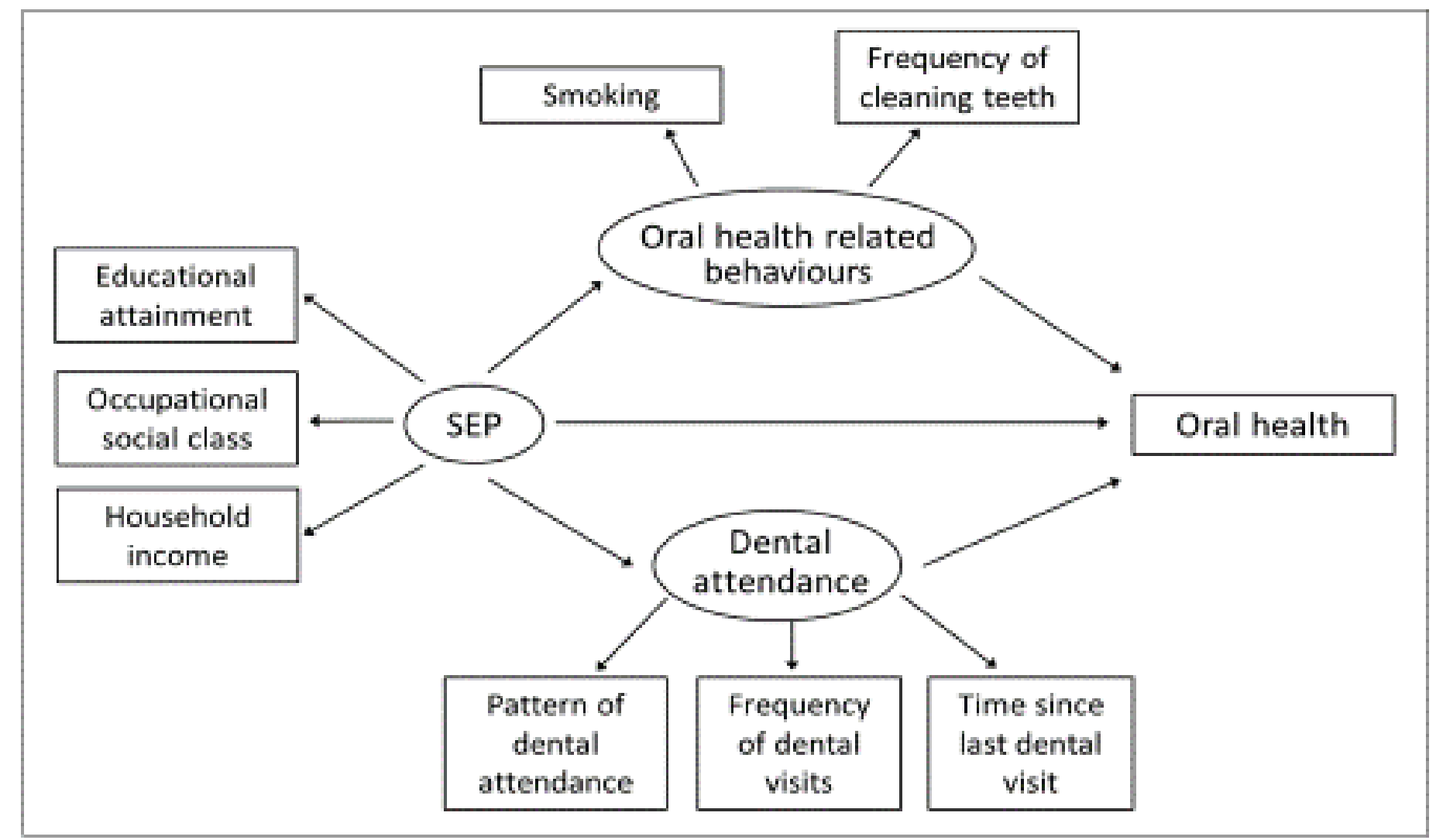

Figure 1 - Path diagram for the conceptual model that guided our analyses. Latent constructs are conventionally depicted by oval shapes.

Note: The model was also adjusted for age 


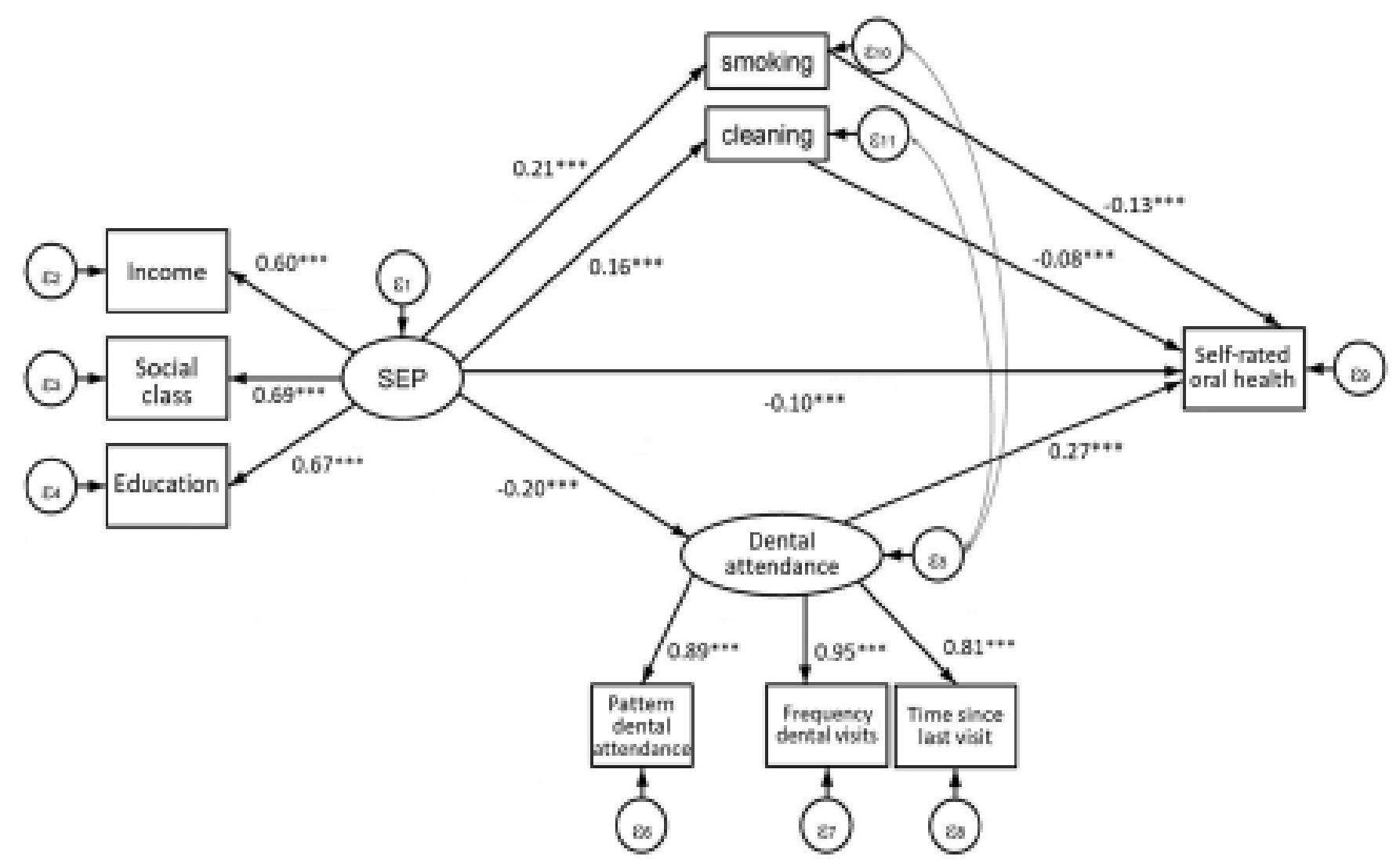

Figure 2 - Path diagram with standardized parameters of the model on self-rated oral health. Model fit statistics: $\mathrm{CFI}=0.972$, RMSEA $=0.040, \mathrm{SRMR}=0.022$. Variables are coded so that high values indicate low SEP, unhealthy behaviours, Bood dental attendance, good self-rated oral health. "* p< $0.01 ; * * * p<0.001$ 


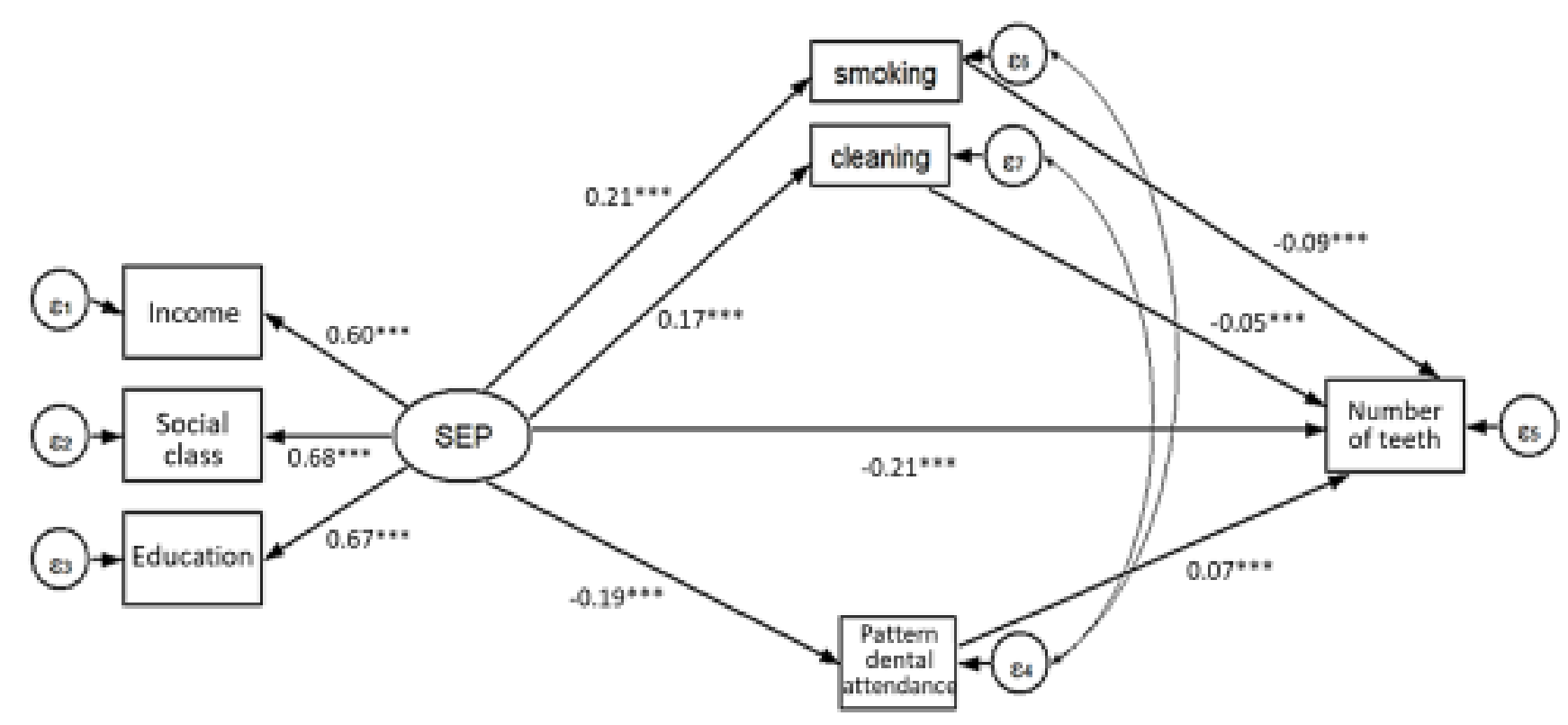

Figure 3 - Path diagram with standardized parameters of the model on number of teeth. Model fit statistics: CFI $=0.961, \mathrm{RMSEA}=0.049 \mathrm{SRMR}=0.027$. Variables are coded so that high values indicate low SEP, unhealthy behaviours, good dental attendance, number of natural teeth. ${ }^{* *} p<0.01 ;{ }^{* * *} p<0.001$ 